\title{
Linear Positional and Speed Control of Servo Carts Using Inverse Dynamic Control
}

\author{
Ibrahim M. Mehedi $\left(\mathbb{D},{ }^{1,2}\right.$ Mohd Heidir Mohd Shah $\mathbb{D D}^{1},{ }^{1}$ and Rahtul Jannat $\mathbb{D D}^{3}$ \\ ${ }^{1}$ Department of Electrical \& Computer Engineering (ECE), King Abdulaziz University, Jeddah, Saudi Arabia \\ ${ }^{2}$ Center of Excellence in Intelligent Engineering Systems (CEIES), King Abdulaziz University, Jeddah, Saudi Arabia \\ ${ }^{3}$ Department of Electrical \& Electronic Engineering (EEE), BRAC University, Dhaka, Bangladesh
}

Correspondence should be addressed to Rahtul Jannat; mrahatuljannat@gmail.com

Received 9 July 2021; Accepted 16 August 2021; Published 25 August 2021

Academic Editor: Dilbag Singh

Copyright ( 92021 Ibrahim M. Mehedi et al. This is an open access article distributed under the Creative Commons Attribution License, which permits unrestricted use, distribution, and reproduction in any medium, provided the original work is properly cited.

\begin{abstract}
Dynamic inverse- (DI-) based control technique has been utilized in many applications and proven to be effective. Recently, the inverse dynamic control (IDC), an expansion to the classical DI technique, has been trending with implementation in many areas. It has been proved that IDC is capable of overcoming some limitations in DI-based techniques, particularly in cancellation of useful nonlinearities. This paper extends the implementation of IDC on the positional and speed control of the linear servo cart system. Simulation results further proves that IDC is an effective and robust controller evidently when comparing it with the proportional velocity and lead compensator controller.
\end{abstract}

\section{Introduction}

The linear servo cart system is used widely in industries especially in manufacturing and automation process. Some of the application example includes pick-and-place system, tool-feeding system in machining process, and indexing of operations like drilling, stamping, and embossing. It became a popular choice in the linear-positioning tool, thanks to the combination of strengths from its actuator and ease of positioning control from servo units. Due to that, various types of controllers have been proposed to improve the positional and speed control of servo systems including the ever-present PID controller and its variances. Although broadly accepted in industries, the traditional PID controllers are known for their poor performance under the influence of disturbances. To tackle this issue, the authors in [1] introduce two-degree-of-freedom PID positional controller where the position reference responses and disturbance responses were allowed to be designed independently, while the authors in [2] combine the application of position interpolation method and modified incremental PID. There were also some other recent works implementing the PID controller on positioning control of servo motors with different tuning methods $[3,4]$.

The functionality of the linear servo cart system involves movement of the mechanical component which can lead to friction resulting in the introduction of highly nonlinear disturbance to the control output. One of the popular methods used to deal with nonlinearity is sliding mode controllers (SMCs). However, a conventional SMC with switching control action suffers a major drawback in the form of chattering. To overcome this weakness, a boundary layer around the switching surface [5] and an integral SMC with switching gains [6] were proposed.

Another common way of dealing with nonlinearities is by employing nonlinear dynamic inversion- (NDI-) based technique as shown in [7]. This type of controller is designed by enforcing a stable linear error dynamics intuitively. It is widely applied especially in aerospace fields such as unmanned combat aerial vehicles (UCAV) [8], pitch axis autopilots [9], and quadrotors [10]. Despite the effectiveness shown by NDI in the presence of disturbances and noises, there is a big limitation in the implementation of such controllers as they are only applicable to the system where 
the model is known accurately. This can be solved using incremental NDI (INDI), an expansion to the classical NDI which only requires a small part of a model to be known. Some implementation of INDI can be found in [11-13].

Inverse dynamic control (IDC) is another expansion of the NDI family where control by inversion of dynamic inverse constraints is achieved through the Moore-Penrose generalized inverse [14]. This technique overcomes many limitations of the classical NDI especially in the cancellation of useful nonlinearities, robustness concerns, and computational challenges arising with square matrix inversion [15]. IDC has been implemented in many applications [16-18]. This paper extends the design and implementation of IDC on the positional and speed control of the linear servo cart system. In this paper, a generalized inverse control technique is demonstrated for Quanser's linear servo cart system in response to other recent advances in modern control techniques, such as adaptive fuzzy control, fractional order control, and inverse control [19-24]. Moreover, by using sensor technology and new materials to optimize concrete maintenance, we can collect necessary data to justify the proposed control law [25-27].

The remainder of this article is organized as follows: The mathematical model of the linear servo cart system is established in Section 2, while Section 3 outlines the IDC design for linear positional control of the linear servo cart system. The technique used to avoid singularity is explained in Section 4, and simulation setup and the results are discussed in Section 5 before this paper is concluded in Section 6.

\section{Mathematical Model of the Linear Servo Cart System}

Figure 1 shows the linear servo cart system. The relationship between the force applied to the cart by the DC motor and resultant motion of the cart can be derived by applying Newton's second law of motion and D'Alembert's principle to the system, as in

$$
M \dot{v}_{c}(t)+F_{\mathrm{aj}}(t)=F_{c}(t)-B_{\mathrm{eq}} v_{c}(t),
$$

where $M, v_{c}$, and $B_{\text {eq }}$ are the mass of the cart, linear velocity of the cart, and the equivalent viscous damping coefficient, respectively, and $F_{\mathrm{aj}}$ is the armature inertial force due to motor rotation acting on the cart which can be defined as

$$
F_{\mathrm{aj}}=\frac{\eta_{g} K_{g} \tau_{\mathrm{aj}}}{r_{\mathrm{mp}}}
$$

where $\eta_{g}$ is the efficiency of the gear box, $K_{g}$ is the gear ratio, and $\tau_{\mathrm{aj}}$ is the armature inertial torque which can be expressed as

$$
\tau_{\text {aj }}=J_{m} \dot{\omega}_{m}(t) .
$$

The angular velocity of the motor shaft can be translated into linear velocity of the cart with the following equation:

$$
\omega_{m}(t)=\frac{K_{g} v_{c}(t)}{r_{\mathrm{mp}}} .
$$

By substituting (3) and (4), (2) can be rewritten as

$$
F_{\mathrm{aj}}=\frac{\eta_{g} K_{g}^{2} J_{m} \dot{v}_{c}(t)}{r_{\mathrm{mp}}^{2}} .
$$

With that, the force in (1) can now be expressed in terms of the linear velocity of the cart and by considering both the electrical parts and the equation of motion:

$$
J_{\mathrm{eq}} \dot{\nu}_{c}(t)+B_{\mathrm{eq}} v_{c}(t)=A_{m} V_{m}(t)
$$

where

$$
B_{\mathrm{eq}}=\frac{\eta_{g} k_{g}^{2} \eta_{m} k_{t} k_{m}+B_{c} r_{\mathrm{mp}}^{2} R_{m}}{r_{\mathrm{mp}}^{2} R_{m}},
$$

and the actuator gain is

$$
A_{m}=\frac{\eta_{g} k_{g} \eta_{m} k_{t}}{r_{\mathrm{mp}} R_{m}} .
$$

Note that $\eta_{m}$ is the efficiency of the motor, $K_{g}, k_{t}$, and $k_{m}$ are the gear ratio, motor torque constant, and back-emf constant, respectively, and $r_{\mathrm{mp}}$ and $R_{m}$ is the radius of the motor pinion and the motor resistance.

Finally, the equivalent inertia term can be expressed as

$$
J_{\mathrm{eq}}=M_{c}+\frac{\eta_{\mathrm{eq}} k_{g}^{2} J_{m}}{r_{\mathrm{mp}}^{2}} .
$$

\section{Design of IDC Control}

The dynamics of the linear servo cart system can be expressed by rearranging (6) as follows:

$$
\dot{v}_{c}=F+\mathrm{GV}_{m} \text {, }
$$

where $F=-J_{\text {eq }}^{-1} B_{\text {eq }} v_{c}$ and $G=J_{\text {eq }}^{-1} A_{m}$. It is known that the velocity of the cart can be obtained by taking the derivative of its linear position:

$$
\dot{x}_{c}=v_{c} \text {. }
$$

In order to track the linear position of the cart precisely, an error function in the form of squared error function of the actual position, $x_{c}$ from its desired position, $x_{\mathrm{cd}}$ is defined as

$$
\xi_{x_{c}}=n_{1}\left(x_{c}-x_{c d}\right)^{2}=n_{1} e_{x_{c}}^{2} .
$$

Similarly, the error function for linear velocity can be written as

$$
\xi_{v_{c}}=n_{2}\left(v_{c}-v_{\mathrm{cd}}\right)^{2}=n_{2} e_{v_{c}}^{2} .
$$

The constraint linear time-varying ordinary differential equation is established based on the deviation functions. Note that the differential orders are corresponding to the 
relative degree of the deviation functions. The equation takes the following form:

$$
\begin{aligned}
\ddot{\xi}_{x_{c}}+c_{1}(t) \dot{\xi}_{x_{c}}+c_{2}(t) \xi_{x_{c}} & =0, \\
\dot{\xi}_{v_{c}}+c_{3}(t) \xi_{v_{c}} & =0,
\end{aligned}
$$

where $c_{1}, c_{2}$, and $c_{3}$ are coefficients that allow the constraint differential equations as in (14) and (15) to achieve uniform asymptotic stability. Therefore, they must be selected carefully as suggested in [15].

The following can be obtained by taking the derivative from (12) and (13):

$$
\begin{aligned}
& \mathbf{A}=\left[\begin{array}{l}
A_{1} \\
A_{2}
\end{array}\right]=\left[\begin{array}{c}
2 n_{1} e_{x_{c}} G \\
2 n_{2} e_{v_{c}} G
\end{array}\right], \\
& \mathbf{B}=\left[\begin{array}{l}
B_{1} \\
B_{2}
\end{array}\right]=\left[\begin{array}{c}
2 n_{1} e_{x_{c}} \dot{v}_{c d}-2 n_{1} \dot{e}_{x_{c}}^{2}-2 n_{1} e_{x_{c}} F-2 c_{1} n_{1} e_{x_{c}} \dot{e}_{x_{c}}-c_{2} n_{1} e_{x_{c}}^{2} \\
2 n_{2} e_{v_{c}} \dot{v}_{c d}-2 n_{2} e_{v_{c}} F-c_{3} n_{2} e_{v_{c}}^{2}
\end{array}\right] .
\end{aligned}
$$

$$
\dot{u}(t)=-u(t)+\frac{\gamma}{e_{x_{c}}(t)^{2}+e_{v_{c}}(t)^{2}}, \quad u(0)>0 .
$$

The homogeneous part of equation (25) is asymptotically stable, while $\gamma$ in the forcing phrase is a positive real-valued constant. The dynamically scaled generalized inverse (DSGI) is formulated as

$$
\mathbf{A}^{*}=\frac{\mathbf{A}^{\mathbf{T}}}{\left(\mathbf{A}^{\mathbf{T}} \mathbf{A}+u(t)\right)} .
$$

Thus, we can update the IDC-based control input voltage by the following expression:

$$
V_{m}^{*}=\mathbf{A}^{*} B .
$$

Finally, we can update (10) by the following expression:

$$
\dot{v}_{c}=F+G\left(\mathbf{A}^{*} B\right) \text {. }
$$

Note that $\mathbf{A}$ has a dimension of $2 \times 1$, in which the $P$ in (24) will be zero when the property of pseudo-inverse $\mathbf{A}^{+} \mathbf{A}=1$. This will make the null control not useful, and therefore, we will not consider it in our control design.

\section{IDC Singularity Avoidance}

The main trouble with generalized inversion techniques is the singularity which is caused by discontinuities in the MPGI matrix function and eventually leads to the structure to go unbounded. Such happens when the inverted matrix tends to switch its rank.

To overcome this problem, we introduce a dynamic scaling factor which is expanded within MPGI. We denote the scaling factor as $u$ and can be defined as

By putting the time derivatives of the equations in the constraint dynamics as in (14) and (15), equations (16)-(18) can be transformed into their algebraic form shown as

where 


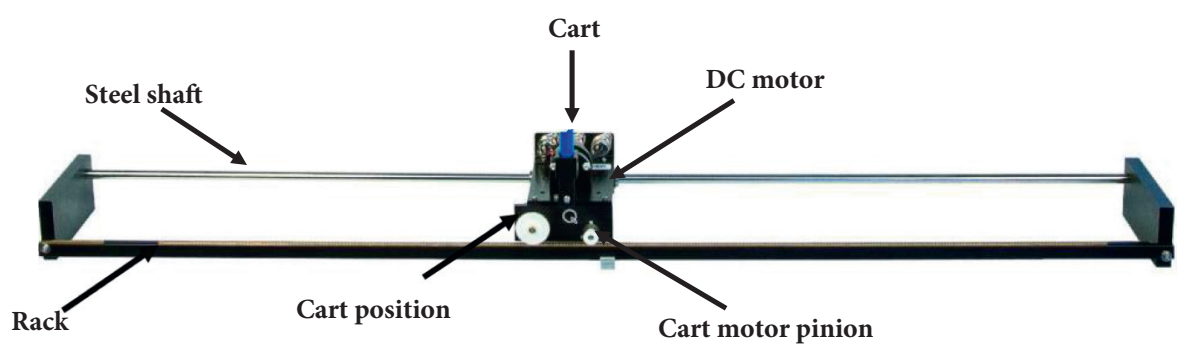

FIgURe 1: Linear servo cart system.

TABle 1: Parameters of the linear servo cart system.

\begin{tabular}{lcc}
\hline Parameters & Values & Units \\
\hline Motor armature resistance, $R_{m}$ & 2.6 & $\Omega$ \\
Rotor moment of inertia, $J_{m}$ & $3.9 \times 10^{-7}$ & $\mathrm{Kgm}^{2}$ \\
Motor current torque constant, $k_{t}$ & $7.7 \times 10^{-3}$ & $\mathrm{Nm} / A$ \\
Motor efficiency, $\eta_{m}$ & 1 & - \\
Motor back-emf constant, $k_{m}$ & $7.7 \times 10^{-3}$ & $\mathrm{~V} /(\mathrm{rad} / \mathrm{s})$ \\
Gear ratio, $k_{g}$ & 3.71 & - \\
Gear efficiency, $\eta_{g}$ & 1 & - \\
Mass of the cart, $M$ & 0.57 & $\mathrm{~kg}$ \\
Motor pinion radius, $r_{\mathrm{mp}}$ & 6.4 & $\mathrm{~mm}$ \\
Equivalent viscous damping coefficient, $B_{\mathrm{eq}}$ & 4.3 & - \\
\hline
\end{tabular}

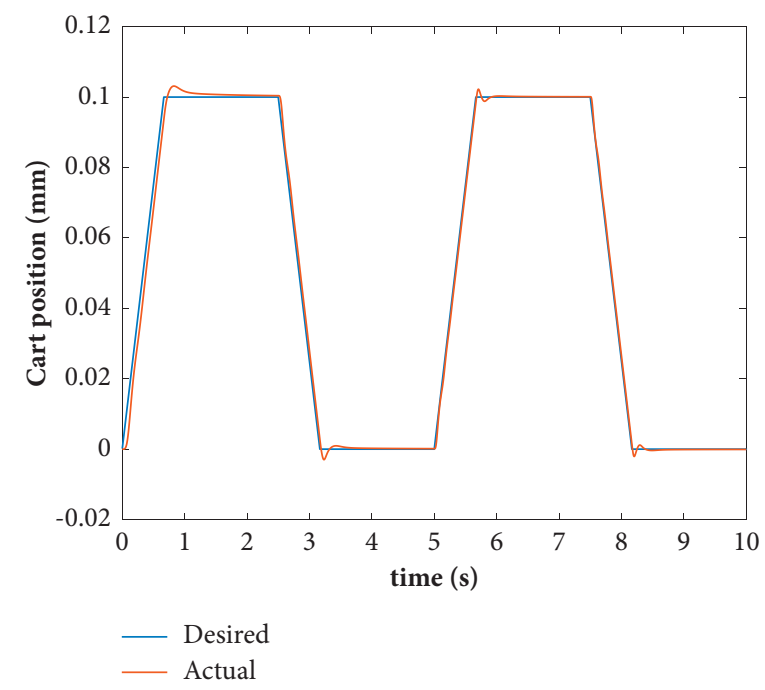

FIgUre 2: Position tracking of the linear servo cart system using IDC.

linear servo cart system parameters, we showed that IDC is able to track the position of the cart according to the profile set accurately as suggested by the actual motion profile in Figure 2 and the squared error norm in Figure 3.

\subsection{Robust Analysis for Sinusoidal Position Tracking. We} evaluate robustness of our designed controller by setting a $0.2 \mathrm{~Hz}$ sinusoidal input reference as shown in Figure 4 having $150 \mathrm{~mm}$ of maximum amplitude with the numerical value of the linear servo cart system changed to $20 \%$ from the one in the previous simulation. For comparison, the system was also simulated using a proportional-velocity controller with proportional gains $K_{p}=274.6159$ and velocity gains $K_{v}=5.5272$. It was obvious from the squared error norm in Figure 5 that IDC outperforms the PV controller in linear position tracking of the linear servo cart system. This simulation results also suggest that IDC is a robust controller.

5.3. Linear Speed Tracking. To evaluate performance of IDC on tracking the speed of the linear servo cart system, we simulate the scenario by giving a $0.25 \mathrm{~Hz}$ square wave set point with an amplitude of $0.2 \mathrm{~m} / \mathrm{s}$. Another simulation is done using a lead compensator (LC) for comparison, and the 


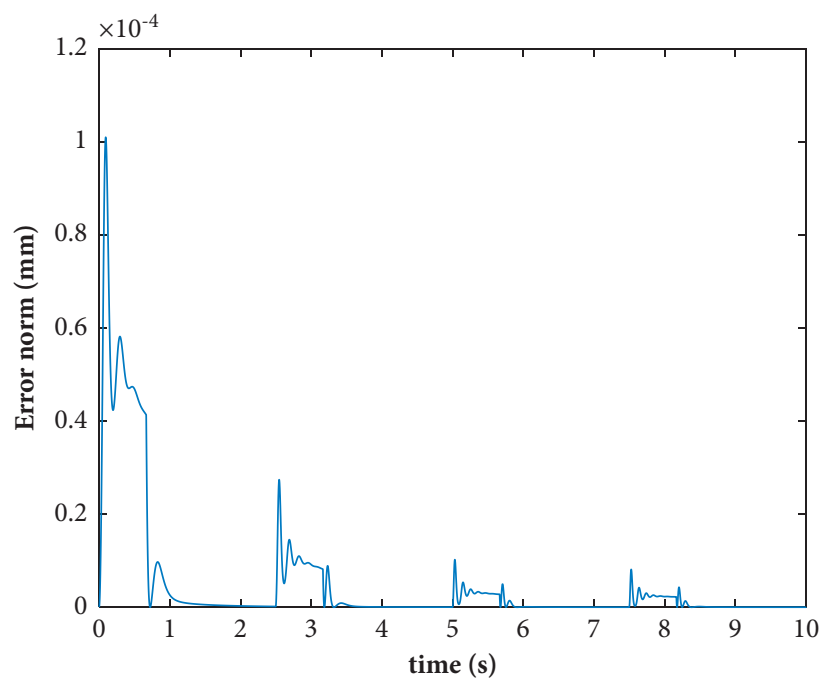

FIGURE 3: Squared error norm for position tracking.

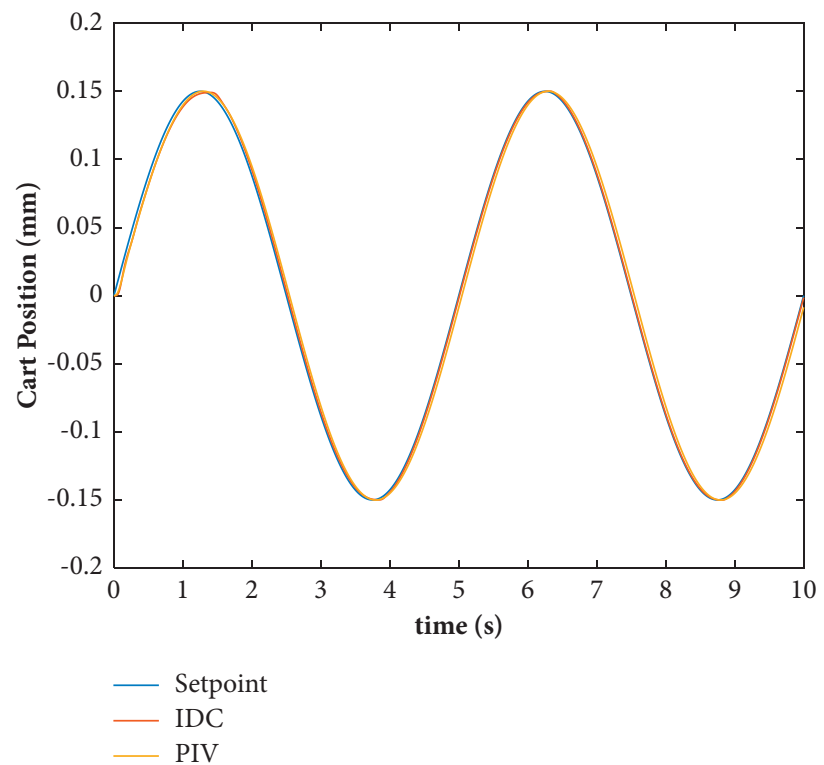

FIgURE 4: Sinusoidal position tracking of the linear servo cart system.

results are as shown in Figure 6. We can see from the simulation results that IDC has the ability to track the linear servo cart's speed smoothly without overshooting. The squared error norm measured from both controllers also suggests that the IDC perform slightly better than the LC controller as shown in Figure 7. 


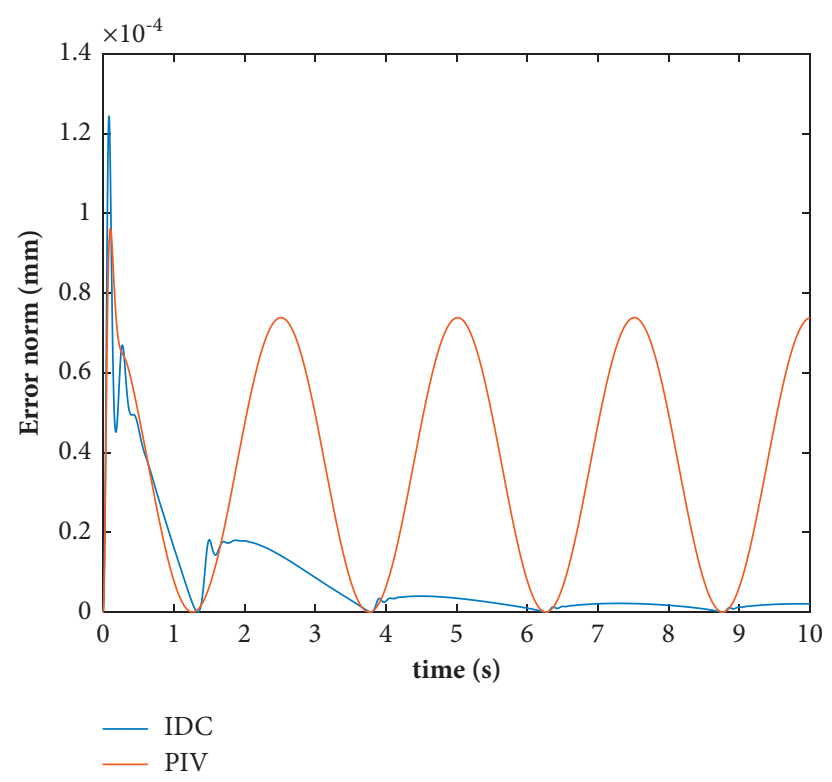

FIGURE 5: Squared error norm for sinusoidal position tracking.

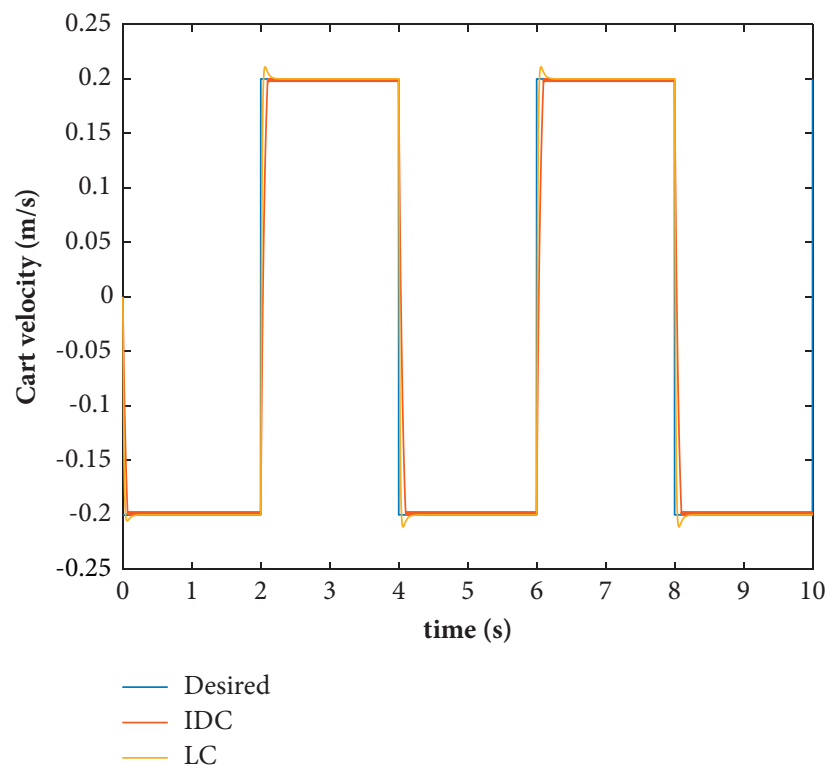

FIgURE 6: Speed tracking of the linear servo cart system. 


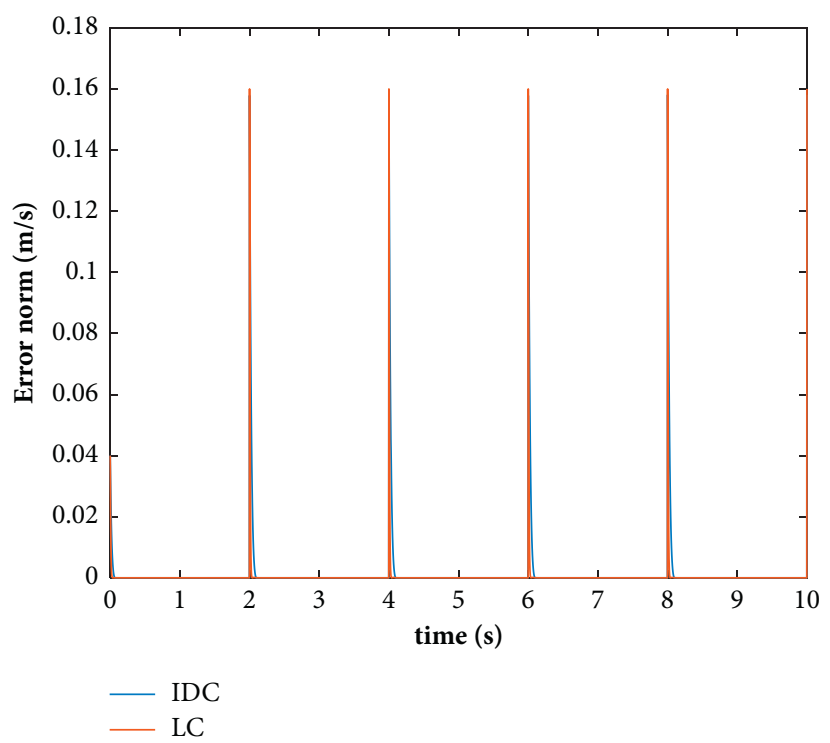

FIGURE 7: Squared error norm for speed tracking.

\section{Conclusions}

An IDC has been successfully designed to control linear position and speed of a linear servo cart system. The controller design has been discussed in detail, particularly in setting up dynamic constraints in the form of constraint differential equations. We have shown that the control law of stable linear position and speed tracking is achieved by inverting the constraint differential equations using MPGI. Through simulations, we have demonstrated that the IDC is an effective and robust controller with performance of tracking linear position and speed of the linear servo cart system, surpassing the performance of PV and LC controllers.

\section{Data Availability}

No data were used to support this study.

\section{Conflicts of Interest}

The authors declare that they have no conflicts of interest.

\section{Acknowledgments}

This research work was funded by Institutional Fund Projects under grant no (IFPRC-040-135-2020). Therefore, the authors gratefully acknowledge technical and financial support from the Ministry of Education and King Abdulaziz University, Jeddah, Saudi Arabia. Furthermore, the authors thank Dr. Salmiah Ahmad of the Department of Mechatronics Engineering at the International Islamic University Malaysia in Kuala Lumpur, Malaysia, for her critical review of the manuscript during submission.

\section{References}

[1] S. Yamamoto, M. Sugiura, J. Sawaki, and K. Matsuse, "Method controller of two-degree-of-freedom for linear servo motor PID position drives," IEEE Transactions on Industry Applications, vol. 116, no. 8, 1996.

[2] Y. Liu, X. Gao, and X. Yang, "Research of control strategy in the large electric cylinder position servo system," Mathematical Problems in Engineering, vol. 2015, Article ID 167628, 2015.

[3] N. S. Rathore and V. P. Singh, "Tuning of PID controller for position control of DC servo motor using luus-jaakola optimization," in Proceedings of the 2015 International Conference on Computer, Communication and Control, pp. 1-5, Indore, India, September 2015.

[4] S. Shrivastava, V. P. Singh, R. K. Dohare, S. P. Singh, and D. P. S. Chauhan, "PID tuning for position control of DC servo-motor using TLBO," International Journal of Advanced Technology and Engineering Exploration, vol. 4, no. 27, pp. 23-27, 2017.

[5] Y. K. Tan and S. K. Panda, "Sliding-mode position controller for linear permanent magnet brushless DC servo motors," in Proceedings of the The Fifth International Conference on Power Electronics and Drive Systems, 2003. PEDS 2003, vol. 2, pp. 1653-1658, Singapore, November 2003.

[6] G. A. Zarkar and S. S. Sankeshwari, "Simulation of dc servo motor position control," The International Journal of Engineering and Advanced Technology, vol. 7, no. 6, pp. 18821888, 2015.

[7] C. Guarino, L. Bianco, and A. Piazzi, "A servo control system design using dynamic inversion," Control Engineering Practice, vol. 10, pp. 847-855, 2002.

[8] J. O. Pedro, A. Panday, and L. Dala, "A nonlinear dynamic inversion-based neurocontroller for unmanned combat aerial vehicles during aerial refuelling," International Journal of 
Applied Mathematics and Computer Science, vol. 23, no. 1, pp. 75-90, 2013.

[9] G. Papageorgiou and M. Polansky, "Tuning a dynamic inversion pitch axis autopilot using McFarlane-Glover loop shaping," Optimal Control Applications and Methods, vol. 30, no. 3, pp. 287-308, 2009.

[10] A. Das, K. Subbarao, and F. Lewis, "Dynamic inversion of quadrotor with zero-dynamics stabilization," in Proceedings of the 2008 IEEE International Conference on Control Applications, pp. 1189-1194, San Antonio, TX, USA, September 2008.

[11] V. Kampen and D. Visser, "Aircraft fault-tolerant trajectory control using incremental nonlinear dynamic inversion aircraft fault-tolerant trajectory control using incremental nonlinear dynamic inversion," Control Engineering Practice, vol. 57, pp. 126-141, 2016.

[12] Y. Huang, D. M. Pool, O. Stroosma, and Q. P. Chu, "Incremental nonlinear dynamic inversion control for hydraulic hexapod flight simulator motion systems ${ }^{* *}$ the first author is sponsored by Chinese scholarship council," IFAC-PapersOnLine, vol. 50, no. 1, pp. 4294-4299, 2017.

[13] Z. Liu, J. Guo, M. Li, S. Tang, and X. Wang, "Research article VTOL UAV transition maneuver using incremental nonlinear dynamic inversion," International Journal of Aerospace Engineering, vol. 2018, Article ID 6315856, 2018.

[14] A. Ben-Israel and T. N. E. Greville, Generalized Inverses: Theory and Applications, Springer-Verlag, New York, NY, USA, 2003.

[15] I. M. Mehedi, U. Ansari, and U. M. AL-Saggaf, "Three degrees of freedom rotary double inverted pendulum stabilization by using robust generalized dynamic inversion control: design and experiments," Journal of Vibration and Control, vol. 26, no. 23-24, pp. 2174-2184, 2020.

[16] H. An and Q. Wu, "Disturbance rejection dynamic inverse control of air-breathing hypersonic vehicles," Acta Astronautica, vol. 151, pp. 348-356, 2018.

[17] L. Rincon, Y. Kubota, G. Venture, and Y. Tagawa, "Inverse dynamic control via "simulation of feedback control" by artificial neural networks for a crane system," Control Engineering Practice, vol. 94, Article ID 104203, 2020.

[18] M. F. Zaeh and S. .J. Pieczona, “Adaptive inverse control of a galvanometer scanner considering the structural dynamic behavior," CIRP Annals, vol. 67, no. 1, pp. 385-388, 2018.

[19] I. M. Mehedi, "State feedback based fractional order control scheme for linear servo cart system," Journal of Vibroengineering, vol. 20, no. 1, pp. 782-792, 2018.

[20] I. M. Mehedi, U. M. Al-Saggaf, R. Mansouri, and M. Bettayeb, "Stabilization of a double inverted rotary pendulum through fractional order integral control scheme," International Journal of Advanced Robotic Systems, vol. 16, no. 4, pp. 1-9, 2019.

[21] U. M. Al-Saggaf, I. M. Mehedi, R Mansouri, and M Bettayeb, "Fractional order linear ADRC-based controller design for heat-flow experiment," Mathematical Problems in Engineering, vol. 2021, Article ID 7291420, 8 pages, 2021.

[22] I. M. Mehedi, U. M. Al-Saggaf, R. Mansouri, and M. Bettayeb, "State feedback based fractional order control scheme for linear servo cart system," Measurement, vol. 135, pp. 13-22, 2019.

[23] A. I. M. Iskanderani and I. M. Mehedi, "Experimental application of robust and converse dynamic control for rotary flexible joint manipulator system," Mathematical Problems in Engineering, vol. 2021, Article ID 8917134, 9 pages, 2021.

[24] I. M. Mehedi, H. S. M. Shah, U. M. Al-Saggaf, R Mansouri, and M Bettayeb, "Adaptive fuzzy sliding mode control of a pressure-controlled artificial ventilator," Journal of Healthcare Engineering, vol. 2021, Article ID 1926711, 10 pages, 2021.

[25] D. Singh, M. Kaur, and H. Singh, "Remote sensing image fusion using fuzzy logic and gyrator transform," Remote sensing letters, vol. 9, no. 10, pp. 942-951, 2018.

[26] M. Kaur, S. Singh, M. Kaur, and M. Kaur, "A comprehensive study on computational pansharpening techniques for remote sensing images," Mathematical Problems in Engineering, vol. 2021, Article ID 5012496, 17 pages, 2021.

[27] D. Singh and V. Kumar, "Dehazing of remote sensing images using improved restoration model based dark channel prior," The Imaging Science Journal, vol. 65, no. 5, pp. 282-292, 2017. 